\title{
3D simulation of radiographic projections to test and reduce the effect of pelvic tilt on the accuracy of cross-table lateral radiography
}

Jie Xu', Baohua Su², Wenhui Zhang ${ }^{1}$, Hao Sun ${ }^{1}$, Deng Li', Zhiqing Cai', Meiyi Chen', Meiling Qiu ${ }^{1}$ and Ruofan $\mathrm{Ma}^{1 *}$

\begin{abstract}
Background: Cross-table lateral $(\mathrm{CL})$ radiography is a convenient and feasible method to assess cup version angle (VA) after total hip arthroplasty; However, pelvic tilt (PT) may contribute to its measurement inaccuracy. How PT affects $\mathrm{CL}$ radiographic measurements have not been well studied. We sought (1) to determine the effect of the PT on cup version measurement on $\mathrm{CL}$ radiography and (2) to develop a method for reducing measurement errors caused by the PT.

Methods: We used 3D technique to construct standard model and capture CL radiography simulation. A linear regression model was created to analyze the relationship between PT and VA. CL radiography and computed tomography (CT) were performed for the enrolled patients after surgery. The consistency between $\mathrm{CL}$ and $\mathrm{CT}$ measurements were verified by intra-class correlation coefficient (ICC).

Results: There was a high correlation between the VA and PT. For each 1-degree increased in the PT, the VA decreased by $0.76^{\circ}\left(R^{2}=0.995, p<0.001\right)$. Based on the data, we created a corrective formula to convert the radiographic measurements into values approximating the actual VA under a natural pelvic position. The VA measurements corrected by our equation was in high agreement with the CT-measured values with reference to the corresponding PT (ICC $=0.988, p<0.001)$, which was in sharp contrast to that without PT control $(I C C=0.454$, $p=0.203)$.
\end{abstract}

Conclusions: The PT may contribute to cup version measurement inaccuracies on $\mathrm{CL}$ radiography. Our mathematical algorithm can serve as a reliable method to improve the accuracy of $\mathrm{CL}$ radiography.

Keywords: Version angle, Acetabular component, Cross-table lateral radiography, Total hip arthroplasty, Pelvic tilt

\footnotetext{
* Correspondence: maruofan@mail.sysu.edu.cn

'Department of Joint Surgery, Sun Yat-sen Memorial Hospital, Sun Yat-sen University, Guangzhou, China
}

Full list of author information is available at the end of the article

(c) The Author(s). 2020 Open Access This article is licensed under a Creative Commons Attribution 4.0 International License, which permits use, sharing, adaptation, distribution and reproduction in any medium or format, as long as you give appropriate credit to the original author(s) and the source, provide a link to the Creative Commons licence, and indicate if changes were made. The images or other third party material in this article are included in the article's Creative Commons licence, unless indicated otherwise in a credit line to the material. If material is not included in the article's Creative Commons licence and your intended use is not permitted by statutory regulation or exceeds the permitted use, you will need to obtain permission directly from the copyright holder. To view a copy of this licence, visit http://creativecommons.org/licenses/by/4.0/ The Creative Commons Public Domain Dedication waiver (http://creativecommons.org/publicdomain/zero/1.0/) applies to the data made available in this article, unless otherwise stated in a credit line to the data. 


\section{Background}

Malposition of acetabular components during total hip arthroplasty (THA) is known as the major risk factor contributing to complications, such as dislocation [1], impingement [2], and accelerated bearing wear [3]. Accurate assessment of the version angle (VA) of acetabular components is important to predict the potential risk of these complications after THA [1-5]. Computed tomography (CT) is considered the most accurate method for the measurement of cup anteversion; however, cost burden and radiation exposure may limit its application on a routine basis [6-8]. Conversely, plain radiography is still commonly employed in clinical practice, including anteroposterior (AP) radiography and cross-table lateral (CL) radiography.

$C L$ radiography may be a more convenient and feasible method than AP radiography, in which cup version can be directly measured and anteversion and retroversion can be discriminated from each other $[9,10]$. Although it may provide acceptable assessment of acetabular component position, it is not reliable enough for precise measurement [10-12]. The relationship between the pelvic tilt (PT) and cup version measurement is well established. Previous studies have consistently shown that each degree of anterior or posterior tilt of the pelvis will change the cup version measurement by approximately $0.8^{\circ}[13-16]$. Concerns on the influence of pelvic inclination variations caused by flexion of the contralateral hip joint during CL film shooting on the accuracy of cup version measurements have been raised. However, the extent to which the PT affects CL radiographic measurements and how to compensate for this effect have not been well studied.

In this study, we aimed to determine the effect of the PT on cup version measurements on CL radiography and to develop a feasible method for modifying measurement inaccuracies caused by the PT.

\section{Patients and methods \\ 3D model building}

To identify the influence of the PT on the acetabular cup version measurement on CL radiography, we constructed the 3D postoperative models of the pelvis using Geomagic Design X 2016 (Geomagic Inc., Morrisville, North Carolina, USA). Initially, we used a laser equipment to scan the titanium converge acetabular cups with a diameter of 48-52 mm (Trilogy Acetabular Hip System, Zimmer, Warsaw, Indiana, USA) to establish the cup models. A CT scan for pelvis was performed on three healthy female volunteers without hip deformities and previous surgeries. We defined the PT as the angle created by a line running from the first sacral midpoint to the symphysis pubis and a line perpendicular to the horizontal plane in the supine position (Fig. 1a). Normal value is about $50^{\circ} \sim 60^{\circ}$. An inclination of $60^{\circ}$ was set as the natural position of the pelvic models. According to the safe zone described by Lewinnek et al. [1], an anteversion of $15^{\circ}$ and an inclination of $45^{\circ}$ were set as the initial position before the cup models were implanted. After the parameters were settled down, the postoperative hip model was completed by implanting the prostheses into the pelves. On this model, parameters including VA and PT can be set as different unit by intervals of $5^{\circ}$ independently.

\section{Patients}

A total of 50 patients who underwent primary THA for hip degeneration diseases were recruited in this study between July 2019 and December 2019, including 21 men and 29 women with an average age of 56 years (range, 32-78 years) and body mass index of $22.74 \mathrm{~kg} / \mathrm{m}^{2}$ (range, $17.60-24.84 \mathrm{~kg} / \mathrm{m}^{2}$ ). None of the patients had spinal or pelvic surgeries or deformities. The same team of surgeons performed all cementless THAs using a posterolateral approach or a direct anterior approach. All acetabular cups were selected from the Trilogy ${ }^{\bullet}$ Acetabular Hip System and R3 ${ }^{\circ}$ Acetabular Hip System (Smith \& Nephew, Inc., Memphis, Tennessee, USA). This study was approved by the ethics committee of our institution. All patients provided written informed consent.

\section{VA and PT measurement on $\mathrm{CL}$ radiography}

The method of $\mathrm{CL}$ radiography has been described in detail in a previous study [17]. The patients were placed in the supine position with the contralateral hip flexed at $90^{\circ}$ and the contralateral leg placed on a small stand to maintain the position. The radiation beam was positioned parallel to the examination table at $45^{\circ}$ to the long axis of the body, and the radiographic film was positioned perpendicular to the examination table. We performed CL radiography 1 day after surgery for each patient and measure VA using the method introduced by Woo and Morrey [9] (Fig. 1b). After CL radiography was performed, we adjusted the X-ray incidence from $45^{\circ}$ to $90^{\circ}$ to enable the pelvic landmarks displayed completely. Then an additional lateral radiography was obtained to measure the PT. The method used to measure the PT on lateral radiograph was based on the PT definition (Fig. 1c).

To simulate VA measurement on 3D model, we placed 3D model in the supine position, rotated it clockwise to $45^{\circ}$, and centered it on the top of the acetabular cup. Thereafter, the model was projected onto the coronal plane, and a two-dimensional image was captured by screen as a CL film simulation. VA was measured on 3D simulated CL radiographs under different setting values of PT and VA (Fig. 1d). 

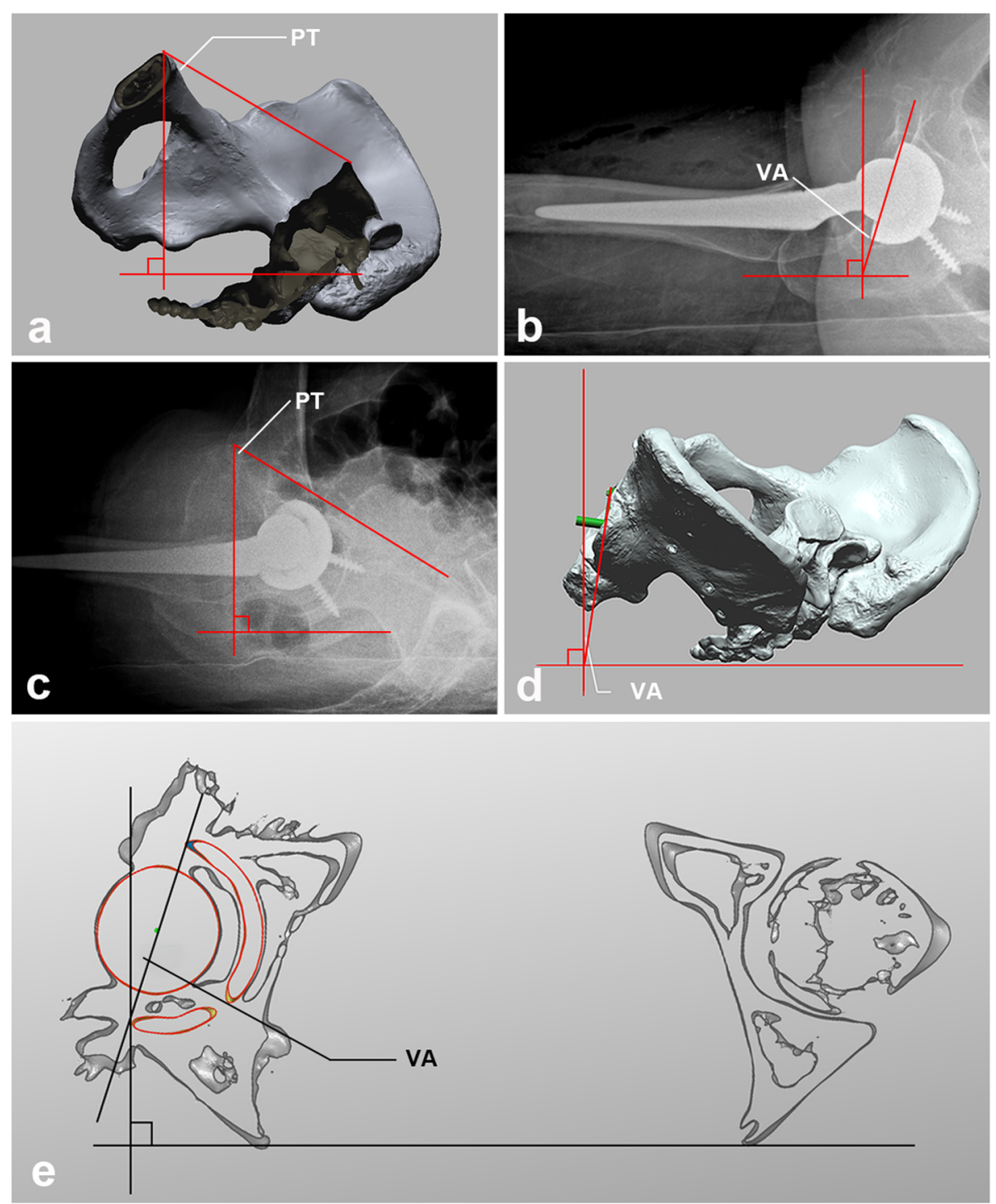

Fig. 1 a Pelvic Tilt in 3D model. Pelvic tilt = the angle created by a line running from the first sacral midpoint to the symphysis pubis and a line perpendicular to the horizontal plane in the supine position. The normal value is about $60^{\circ}$. b Measurement of VA on Cross-table lateral Radiography. VA was measured using the following formula: anteversion = angle between a line along the rim of the cup and a line perpendicular to the horizontal plane. c Measurement of pelvic tilt from the lateral radiograph. $\mathbf{d}$ VA Measurements in 3D Simulation. Cross-table radiography was simulated by capturing a screenshot in the lateral position with rotation angles of $45^{\circ}$. PS. As the edge of the cup was obscured by the acetabulum, we reconstructed an acetabulum axis (the green component in the figure) for convenience and accuracy of measurement. $\mathbf{e}$ VA measurement on the transverse plane of 3D-transformed and PT-adjusted CT with the reference of posterior aspects of the ischium

\section{Consistency verification by $\mathrm{CT}$}

The accuracy and reliability of the measured VA was verified by compared with the $C T$ value. The patients underwent a CT scan for the pelvis 7 days after surgery and were placed in the supine position with the bilateral hip joints in a neutral position. To ensure that the VA measured on CT and CL radiography was compared at a consistent PT, we used Geomagic Wrap 2017 (Geomagic Inc.) to create the $3 \mathrm{D}$ pelvic model based on $\mathrm{CT}$ data so that the PT could be set to a certain value. The VA was measured under the PT set to the measured value on the radiographs to compare the $\mathrm{CL}$ radiographymeasured VA. The method of measuring the VA on CT was referenced to a previous study (Fig. 1e) [10].

\section{Statistical analysis}

Three qualified orthopedic surgeons performed all measurements. The intra-class correlation coefficient (ICC) and $95 \%$ confidence interval were calculated to assess the intra- and inter-observer reliabilities. We used the two-way random effects intra-class correlation model and absolute agreement to calculate the ICC: an ICC of 
1 indicated perfect reliability, while an ICC of 0 indicated the opposite. Linear regression was used to assess the correlation between the PT and acetabular cup anteversion. The ICC was used to determine the validity of the measured VA and corrected VA by comparing them with the corresponding CT values. Statistical analyses were performed using SPSS ver. 22.0 (SPSS Inc., Chicago, Illinois, USA). The significance level was set at $p$-values of $<0.05$.

\section{Results}

\section{Repeatability}

All intra-observer and inter-observer ICCs were excellent for the measurements of the PT obtained from the additional lateral radiographs and VA obtained from the CL radiographs, 3D-simulated CL radiographs, and CT scans (Table 1).

\section{Effect of the PT on VA measurement}

The mean values of the VA under different actual anteversion angles and PTs are shown in Table 2. A significant tendency could be seen from the scatter plot (Fig. 2a) drawn on the basis of the measured error under the PT difference. The measured error decreased along with the PT closer to $60^{\circ}$. A Pearson correlation coefficient of $0.998(p<0.001)$ was obtained in the correlation analysis, indicating a high correlation between the measured error and PT. The linear regression equation was $y=-0.76 x-0.13\left(R^{2}=0.995, p<0.001\right)$, showing that for each 1-degree difference in the PT from the standard value $\left(60^{\circ}\right)$, the measured error increased $\left(0.76^{\circ}\right)$.

\section{Equation derivation procedure}

From the data presented in Table 2, different linear correlations between the PT and measurement under the different actual VAs are shown in Fig. 2b. By integrating the different linear correlations, we used eq. 1 to represent the gross linear correlations between the PT and measurement; $b$ was defined as the intercept on the $y$-axis. We established a mathematical correlation (eq. 2) between the intercept of the lines representing the different actual VAs on the $y$-axis and the corresponding actual VA. Finally, the two equations were integrated and transformed to obtain eq. 3 , which could be applied to convert the VA into the actual value in the 3Dsimulated CL radiograph under the PT of $60^{\circ}$.

$$
\begin{aligned}
& V A_{m}=0.76 \times P T+b \\
& b=0.99 \times V A+45.49 \\
& V A=\frac{0.76 \times P T+V A_{m}-45.49}{0.99}
\end{aligned}
$$

$V A_{m}:$ Measurement of the acetabular cup version angle. VA: Actual acetabular cup version angle set in three-dimensional simulation. $b$ : $y$-intercept

\section{Accuracy and agreement}

The consistency test indicated that both the measured value of the VA $(\mathrm{ICC}=0.997, p<0.001)$ and its formula-converted value ( $\mathrm{ICC}=0.988, p<0.001$ ) were in high agreement with the CT-measured value adjusted by the corresponding PT. In contrast, the consistency between the anteversion measurement and CT measurement without PT adjustment was poor (ICC $=0.454, p=0.203)$.

\section{Discussion}

In the present study, we established 3D postoperative models for CL radiograph simulation and data analysis. The advantages are that each variable can be set independently within a wide range, and confounding factors can be effectively controlled. The analysis showed that the PT had a significant effect on the measurement of the VA on CL radiographs. The formula we derived can convert the measured values under different PTs into actual values under the natural pelvic position. After comparing the corrected values from the clinical measurement data with the $\mathrm{CT}$ values, we found that they were highly consistent, which confirmed the high fidelity and reliability of the 3D simulation method and inferred formula. Our correction approach can effectively improve the accuracy of $\mathrm{CL}$ radiography when measuring the VA.

Table 1 Reliability of All Measurements

\begin{tabular}{lll}
\hline & ICC for Intra-Observer $(\mathbf{9 5 \%} \mathrm{Cl})$ & ICC for Inter-Observer $(\mathbf{9 5 \%} \mathrm{Cl})$ \\
\hline $\mathrm{PT}$ & $0.962(0.946$ to 0.987$)$ & $0.933(0.899$ to 0.963$)$ \\
$\mathrm{VCL}$ & $0.938(0.891$ to 0.963$)$ & $0.923(0.897$ to 0.941$)$ \\
$\mathrm{VCL}_{3 \mathrm{D}}$ & $0.953(0.935$ to 0.968$)$ & $0.962(0.946$ to 0.987$)$ \\
$\mathrm{VCT}_{0}$ & $0.982(0.968$ to 0.989$)$ & $0.936(0.916$ to 0.958$)$ \\
$\mathrm{VCT}_{1}$ & $0.948(0.968$ to 0.989$)$ & $0.925(0.889$ to 0.953$)$ \\
\hline
\end{tabular}

ICC, intraclass correlation coefficient. Cl, 95\% confidence interval. Mean (95\% confidence interval). PT, pelvic tilt measured on lateral radiographs. VCL, version angle measured on $\mathrm{CL}$ radiographs. $\mathrm{VCL}_{3 \mathrm{D}}$, version angle measured on 3-D simulated $\mathrm{CL}$ radiographs under different actual VA and $\mathrm{PT}$. VCT measured on CT without adjusted the pelvic tilt. $\mathrm{VCT}_{1}$, version angle measured on CT which was converted into 3-D model and adjusted PT to the value measured on radiographs 
Table 2 Mean measured values under different PTs \& actual VAs

\begin{tabular}{|c|c|c|c|c|c|c|c|c|c|}
\hline \multirow{2}{*}{$\begin{array}{l}\text { Pelvic } \\
\text { Tilt }\end{array}$} & \multicolumn{9}{|c|}{ Actual VA } \\
\hline & $\overline{0^{\circ}}$ & $5^{\circ}$ & $10^{\circ}$ & $15^{\circ}$ & $20^{\circ}$ & $25^{\circ}$ & $30^{\circ}$ & $35^{\circ}$ & $40^{\circ}$ \\
\hline $20^{\circ}$ & 29.60 & 34.41 & 39.44 & 44.59 & 49.34 & 54.06 & 59.82 & 64.84 & 70.05 \\
\hline $25^{\circ}$ & 26.03 & 30.62 & 35.71 & 40.65 & 46.87 & 51.18 & 56.69 & 61.68 & 66.16 \\
\hline $30^{\circ}$ & 22.57 & 27.63 & 32.17 & 37.99 & 43.02 & 48.65 & 52.39 & 57.50 & 63.23 \\
\hline $35^{\circ}$ & 18.70 & 23.90 & 29.60 & 34.20 & 39.42 & 44.09 & 49.58 & 54.92 & 59.33 \\
\hline $40^{\circ}$ & 15.30 & 20.49 & 25.53 & 31.32 & 35.86 & 40.44 & 45.00 & 50.54 & 55.93 \\
\hline $45^{\circ}$ & 11.39 & 16.54 & 21.55 & 26.41 & 32.22 & 36.80 & 41.22 & 47.02 & 50.26 \\
\hline $50^{\circ}$ & 8.68 & 12.83 & 17.48 & 21.84 & 27.24 & 31.73 & 37.69 & 42.36 & 48.03 \\
\hline $55^{\circ}$ & 6.66 & 5.48 & 12.98 & 18.72 & 23.28 & 28.39 & 33.58 & 38.12 & 43.66 \\
\hline $60^{\circ}$ & 0.66 & 4.11 & 7.34 & 13.45 & 18.76 & 24.79 & 30.86 & 35.59 & 39.83 \\
\hline $65^{\circ}$ & -2.76 & 1.32 & 3.84 & 11.21 & 15.51 & 20.37 & 26.16 & 31.80 & 36.12 \\
\hline $70^{\circ}$ & -9.40 & -1.83 & 0.82 & 3.59 & 11.45 & 17.47 & 23.34 & 27.89 & 32.87 \\
\hline $75^{\circ}$ & -12.75 & -6.13 & 2.31 & 2.64 & 6.78 & 13.46 & 19.55 & 24.85 & 29.52 \\
\hline $80^{\circ}$ & -16.70 & -11.63 & -4.16 & 0.80 & 4.05 & 9.41 & 14.44 & 20.55 & 25.81 \\
\hline
\end{tabular}

This study has several limitations. First, the data in our study were obtained from 3D models based on cases of a non-deformed pelvis because we sought to avoid the interference of other confounding factors (such as osteophytes and deformities) when determining the effects of the PT. The findings may not be suitable for abnormal cases. Second, we used the outline of the 3D images to replace the actual radiographs, enabling the setting of variables to be more accurate and extensive; however, the magnification of the radiographic edge cannot be well simulated, although it will not have a great impact on the measurement of the central visual field.

Reports in the literature with regard to the accuracy of CL radiographic measurement compared with that of CT measurement are conflicting $[6,11,18,19]$. Some investigators found that the CL radiographic value was smaller than the CT value $[11,19]$, while others revealed the opposite $[6,18]$. However, no control or influencing factors, such as the PT, was examined in those studies. The current study bridged the gap and identified the significant implications of the PT on the accuracy of CL radiographic measurement. When the $\mathrm{PT}$ changed by $1^{\circ}$, the measurement error could reach $0.76^{\circ}$. If the PT deviates significantly from the norm, the error may be considerable. Unfortunately, during the course of CL film imaging, the flexion of the lower contralateral extremities to avoid occlusion would lead to variations in the $\mathrm{PT}$, which would be more pronounced in patients with contralateral hip stiffness. Moreover, for CT measurement, owing to the factors of anatomical specificity,

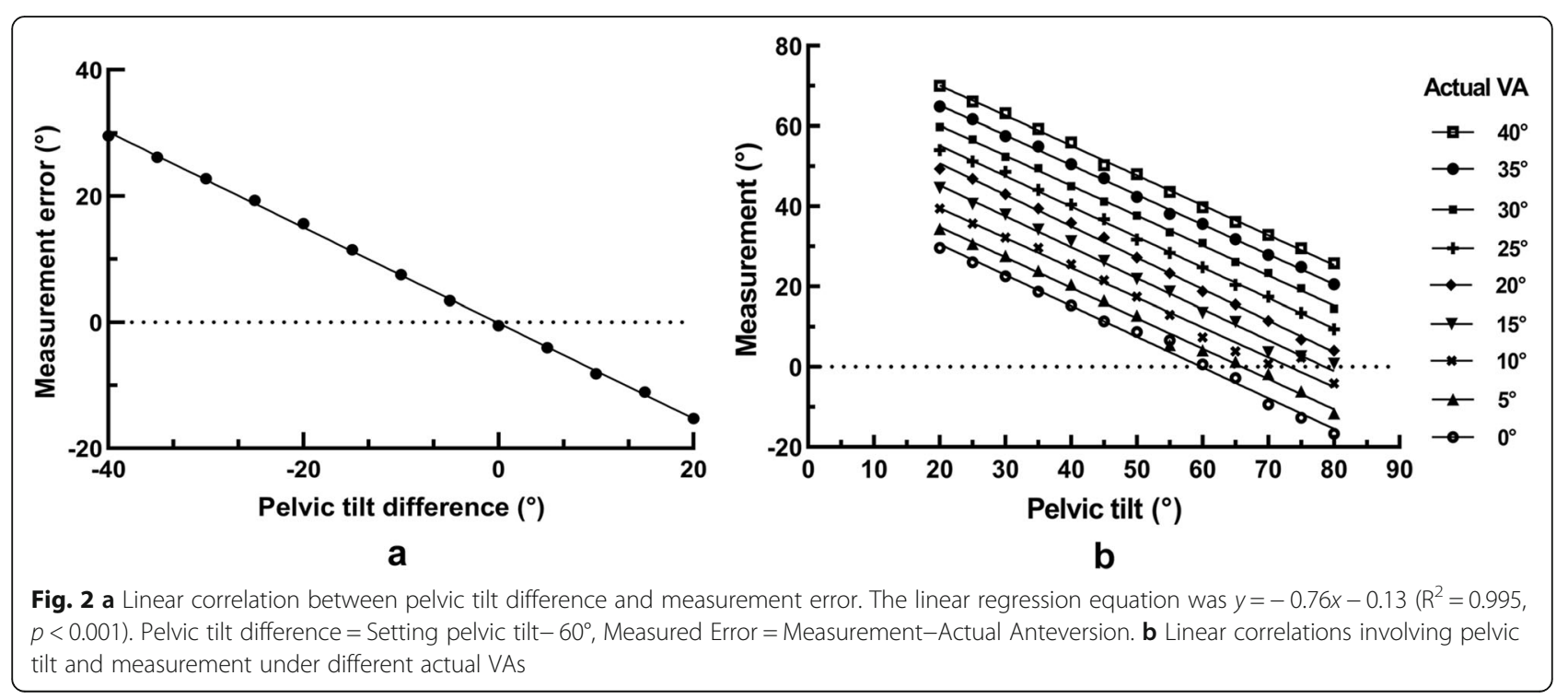


pathological changes, and poor posture, there will inevitably be potential differences in the PT among different patients. When the PT was balanced between the $\mathrm{CL}$ radiographic and CT measurements, we were pleasantly surprised to find that the two measurements were highly consistent (ICC $=0.997, p<0.001$ ); however, these were in sharp contrast to those without control $(\mathrm{ICC}=0.454$, $p=0.203$ ). Accordingly, we believe that the PT is the main factor affecting the accuracy of CL radiographic measurement.

To reduce this effect, we derived a correction formula, which can convert the measured values under different PTs into the actual VA values under the natural pelvis, and it was verified to be reliable after comparison with the CT values. Several methods have been described for correcting the error of VA measurement owing to the PT in previous studies $[15,20,21]$. Some of them used formulae as we did $[15,20]$, while others modified methods using special techniques, such as normograms [21]. However, these methods are relatively complex and cumbersome. In contrast, our method can achieve reliable correction only through a simple equation.

Nevertheless, both the definition of the safety zone and the correction method were evaluated on the basis of the supine position. It has been reported that there are different changes in acetabular cup anteversion in different positions. Lazennec et al. [22] performed a CT scan in the supine and standing positions in patients undergoing hip replacement, finding that the VA significantly increased in the standing position because of the change in the included angle between the patient functional plane and the APP, which exceeded the limits of the safe zone defined by Lewinnek et al. [1] Therefore, only evaluating the acetabular component anteversion in the supine position is not sufficient. Mathematical algorithm may be employed to solve this problem. For instance, a patient was selected to receive $\mathrm{CL}$ radiography and additional lateral radiography to measure the VA and PT in the supine position 1 day after THA. One more lateral radiography at the standing position was performed on this patient to measure the PT 1 month after surgery. Through our equation, the actual VA in the standing position was calculated. If we include a few more patients for analysis, a new formula between the PT and VA in the standing position would be developed, indicating that by obtaining only a lateral film to measure the PT in the standing position, we can determine the actual VA using the equation. Thus, our next study is aimed at determining whether potential mathematical correlation could be explored to calculate actual acetabular anteversion in the standing position, making it possible to more easily and precisely assess the position of the acetabular cup after THA.

\section{Conclusions}

For avoiding the occurrence of adverse events after THA, a simple, accurate, and cheap assessment for cup version is necessary. CL radiography is a potentially eligible method but may present certain errors owing to variations in the PT. For each 1-degree change in the $\mathrm{PT}$, the measurement error can reach $0.76^{\circ}$. However, after correction for the PT using our equation, the measured value can be converted to the approximate anatomical anteversion value under the natural pelvic position, which would be in high agreement with the CT value. Our mathematical algorithm can serve as a reliable method to improve the accuracy of CL radiography.

\section{Abbreviations}

CL: radiography Cross-table lateral radiography; VA: Version angle; THA: Total hip arthroplasty; PT: Pelvic tilt; CT: Computed tomography; AP

radiography: Anteroposterior radiography; ICC: Intra-class correlation coefficient

\section{Acknowledgments}

The research team thanks Guangzhou Jeatech Co., Ltd. for their help in the study coordination.

\section{Authors' contributions \\ JX Conceptualization, Writing manuscript, Project administration. BHS Methodology, Investigation, Writing manuscript. WHZ Methodology, Investigation, Data Curation, Validation. HS Methodology, Investigation. DL Investigation. ZQC Investigation. MYC Formal analysis, Visualization. MLQ Resources. RFM Conceptualization, Supervision. The first two authors contributed equally to this manuscript. All authors read and approved the final manuscript.}

\section{Funding}

This study was supported by Guangzhou Science, Technology and Innovation Commission (201803010011), Natural Science Foundation of Guangdong Province (2019A1515011647), Natural Science Foundation of Guangdong Province (2018A030310231), Fundamental Research Funds for the Central Universities (CN)(18zxxt61).

\section{Availability of data and materials}

The datasets during and/or analysed during the current study available from the corresponding author on reasonable request.

\section{Ethics approval and consent to participate}

All experimental procedures were approved by the ethical committee of Sun Yat-sen Memorial Hospital of Sun Yat-sen University. All volunteers included in the study provided written informed consent.

\section{Consent for publication}

Not applicable.

\section{Competing interests}

On behalf of all authors, the corresponding author states that there is no conflict of interest.

\section{Author details}

'Department of Joint Surgery, Sun Yat-sen Memorial Hospital, Sun Yat-sen University, Guangzhou, China. ${ }^{2}$ Department of Orthopedic Surgery, the Third Affiliated Hospital of Guangzhou Medical University, Guangzhou, China. 
Received: 29 June 2020 Accepted: 3 December 2020

Published online: 14 December 2020

\section{References}

1. Lewinnek GE, Lewis $\mathrm{L}$, Tarr R, Compere CL, Zimmerman JR. Dislocations after total hip-replacement arthroplasties. J Bone Joint Surg Am. 1978;60(2): 217-20.

2. Yoshimine F. The safe-zones for combined cup and neck anteversions that fulfill the essential range of motion and their optimum combination in total hip replacements. J Biomech. 2006;39(7):1315-23.

3. Wan Z, Boutary M, Dorr LD. The influence of Acetabular component position on Wear in Total hip Arthroplasty. J Arthroplast. 2008;23(1):51-6.

4. Danoff JR, Bobman JT, Cunn G, Murtaugh T, Gorroochurn P, Geller JA, Macaulay W. Redefining the Acetabular component safe zone for posterior approach Total hip Arthroplasty. J Arthroplast. 2016:31(2):506-11.

5. Dargel J, Oppermann J, Bruggemann GP, Eysel P. Dislocation following total hip replacement. Dtsch Arztebl Int. 2014;111(51-52):884-90.

6. Ghelman B, Kepler CK, Lyman S, Della Valle AG. CT outperforms radiography for determination of acetabular cup version after THA. Clin Orthop Relat Res. 2009;467(9):2362-70.

7. Komeno M, Hasegawa M, Sudo A, Uchida A. Computed tomographic evaluation of component position on dislocation after total hip arthroplasty. Orthopedics. 2006;29(12):1104-8.

8. Jaramaz B, DiGioia AM 3rd, Blackwell M, Nikou C. Computer assisted measurement of cup placement in total hip replacement. Clin Orthop Relat Res. 1998:354:70-81.

9. Woo RY, Morrey BF. Dislocations after total hip arthroplasty. J Bone Joint Surg Am. 1982;64(9):1295-306.

10. Nunley RM, Keeney JA, Zhu J, Clohisy JC, Barrack RL. The reliability and variation of acetabular component anteversion measurements from crosstable lateral radiographs. J Arthroplast. 2011;26(6 Suppl):84-7.

11. Reikeras $\mathrm{O}$, Gunderson RB. Cross table lateral radiography for measurement of acetabular cup version. Ann Transl Med. 2016:4(9):169.

12. McArthur B, Cross M, Geatrakas C, Mayman D, Ghelman B. Measuring acetabular component version after THA: CT or plain radiograph? Clin Orthop Relat Res. 2012;470(10):2810-8.

13. Malik A, Wan Z, Jaramaz B, Bowman G, Dorr LD. A validation model for measurement of acetabular component position. J Arthroplast. 2010;25(5): 812-9.

14. van Bosse HJ, Lee D, Henderson ER, Sala DA, Feldman DS. Pelvic positioning creates error in CT acetabular measurements. Clin Orthop Relat Res. 2011; 469(6):1683-91.

15. Lembeck B, Mueller $O$, Reize $P$, Wuelker N. Pelvic tilt makes acetabular cup navigation inaccurate. Acta Orthop. 2005;76(4):517-23.

16. Maratt JD, Esposito Cl, McLawhorn AS, Jerabek SA, Padgett DE, Mayman DJ. Pelvic tilt in patients undergoing total hip arthroplasty: when does it matter? J Arthroplast. 2015;30(3):387-91.

17. Nishino H, Nakamura S, Arai N, Matsushita T. Accuracy and precision of version angle measurements of the acetabular component after total hip arthroplasty. J Arthroplast. 2013;28(9):1644-7.

18. Nomura T, Naito M, Nakamura Y, Ida T, Kuroda D, Kobayashi T, Sakamoto T, $\mathrm{Seo} \mathrm{H}$. An analysis of the best method for evaluating anteversion of the acetabular component after total hip replacement on plain radiographs. Bone Joint J. 2014;96-b(5):597-603.

19. Noback PC, Danoff JR, Herschmiller T, Bobman JT, Shah RP, Geller JA, Macaulay W. Plain radiographs are a useful substitute for computed tomography in evaluating Acetabular cup version. J Arthroplast. 2016;31(10): 2320-4

20. Schwarz TJ, Weber M, Dornia C, Worlicek M, Renkawitz T, Grifka J, Craiovan B. Correction of pelvic tilt and pelvic rotation in cup measurement after THA - an experimental study. Rofo. 2017;189(9):864-73.

21. Babisch JW, Layher F, Amiot LP. The rationale for tilt-adjusted acetabular cup navigation. J Bone Joint Surg Am. 2008;90(2):357-65.

22. Lazennec JY, Thauront F, Robbins CB, Pour AE. Acetabular and femoral Anteversions in standing position are outside the proposed safe zone after Total hip Arthroplasty. J Arthroplast. 2017;32(11):3550-6.

\section{Publisher's Note}

Springer Nature remains neutral with regard to jurisdictional claims in published maps and institutional affiliations.

Ready to submit your research? Choose BMC and benefit from:

- fast, convenient online submission

- thorough peer review by experienced researchers in your field

- rapid publication on acceptance

- support for research data, including large and complex data types

- gold Open Access which fosters wider collaboration and increased citations

- maximum visibility for your research: over $100 \mathrm{M}$ website views per year

At BMC, research is always in progress.

Learn more biomedcentral.com/submissions 